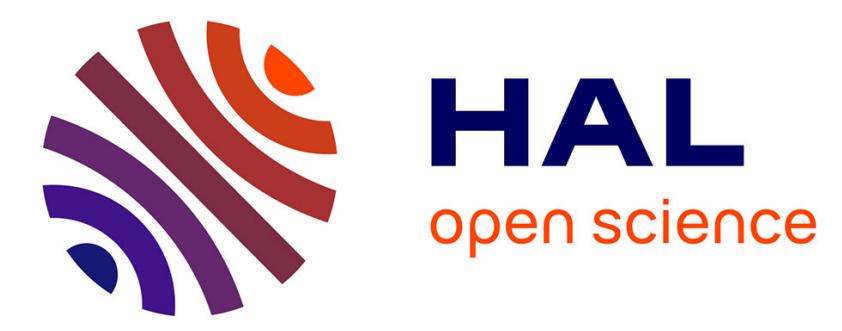

\title{
A Metrological Framework For Hyperspectral Texture Analysis Using Relative Spectral Difference Occurrence Matrix
}

Rui Jian Chu, Noël Richard, Faouzi Ghorbel, Christine Fernandez-Maloigne, Jon Yngve Hardeberg

\section{To cite this version:}

Rui Jian Chu, Noël Richard, Faouzi Ghorbel, Christine Fernandez-Maloigne, Jon Yngve Hardeberg. A Metrological Framework For Hyperspectral Texture Analysis Using Relative Spectral Difference Occurrence Matrix. 2019 10th Workshop on Hyperspectral Imaging and Signal Processing: Evolution in Remote Sensing (WHISPERS), Sep 2019, Amsterdam, Netherlands. pp.1-5, 10.1109/WHISPERS.2019.8921335 . hal-02481582

\section{HAL Id: hal-02481582 https://hal.science/hal-02481582}

Submitted on 17 Feb 2020

HAL is a multi-disciplinary open access archive for the deposit and dissemination of scientific research documents, whether they are published or not. The documents may come from teaching and research institutions in France or abroad, or from public or private research centers.
L'archive ouverte pluridisciplinaire HAL, est destinée au dépôt et à la diffusion de documents scientifiques de niveau recherche, publiés ou non, émanant des établissements d'enseignement et de recherche français ou étrangers, des laboratoires publics ou privés. 


\title{
A METROLOGICAL FRAMEWORK FOR HYPERSPECTRAL TEXTURE ANALYSIS USING RELATIVE SPECTRAL DIFFERENCE OCCURRENCE MATRIX
}

\author{
Rui Jian Chu ${ }^{a}$, Noel Richard ${ }^{a}$, Faouzi Ghorbel $^{b}$, Christine Fernandez-Maloigne $^{a}$, Jon Yngve Hardeberg ${ }^{c}$ \\ ${ }^{a}$ XLIM Laboratory, JRU CNRS 7252, University of Poitiers, France \\ ${ }^{b}$ CRISTAL Laboratory, Ecole Nationale des Sciences de l'Informatique (ENSI), Tunisia \\ ${ }^{c}$ Department of Computer Science, Norwegian University of Science and Technology, Norway
}

\begin{abstract}
A new hyperspectral texture descriptor, Relative Spectral Difference Occurrence Matrix (RSDOM) is proposed. Developed in a metrological framework, it simultaneously considers the distribution of spectra and their spatial arrangement in the hyperspectral image. It is generic and adapted for any number of spectral band or range. As validation, a texture classification scheme is applied on HyTexiLa dataset using RSDOM. The obtained accuracy is excellent (95.6\%), comparable to Opponent Band Local Binary Pattern (OBLBP) but at a much-reduced feature size (0.1\% of OBLBP's).
\end{abstract}

Index Terms - Texture, non-uniformity, hyperspectral, metrology, Kullback-Leibler divergence

\section{INTRODUCTION}

The meteoric rise of spatial resolution of future hyperspectral sensors for Earth Observation (EO) applications (e.g. 30 $\mathrm{m}$ for HISUI, $10 \mathrm{~m}$ for SHALOM, $8 \mathrm{~m}$ for HypXIM) [1] accelerates the incorporation of textural information in aerial image analysis. In cases where recognition based on spectral information alone is insufficient due to the observation being spectrally heterogeneous, textural assessment provides complementary knowledge for land classification or analysis [2]. It allows quantification of various spatial aspects such as directionality, granularity and regularity which further improves discrimination. Such application can be found in a plethora of literature, for instances classification of tree species [3], soil texture estimation [4] and urban landscape identification [5].

Recently, metrology or science of measurement has received considerable attention in the field of remote sensing $[6,7,8]$. Due to the increasing impact on national defense, environmental research and navigation, it becomes critical to impose metrology starting from acquisition steps up to the final data analysis. Consequently, the complete physical meaning of the measurement data has to be preserved throughout the processing for metrological traceability. This is to ensure

This work is supported by the French national projects ANR DigiPi and ERDF NUMERIC / e-Patrimoine.

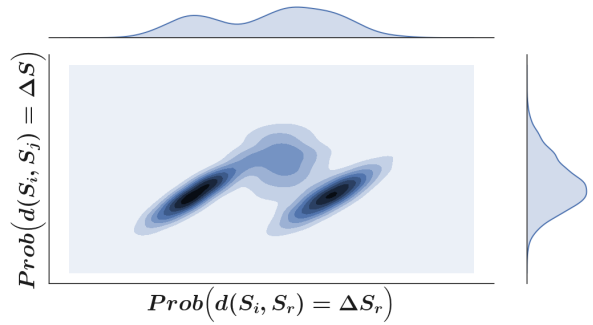

Fig. 1: A conceptual illustration of RSDOM as joint probability distribution of the spectral distribution $\operatorname{Prob}\left(d\left(S_{i}, S_{r}\right)=\right.$ $\left.\Delta S_{r}\right)$ and their spatial arrangement $\operatorname{Prob}\left(d\left(S_{i}, S_{j}\right)=\Delta S\right)$.

that the "result can be related to a reference through a documented unbroken chain of calibrations, each contributing to the measurement uncertainty" [9]. This warrants reproducibility of results and generates confidence in its implication. In the context of our work, this induces a need to define a hyperspectral texture descriptor that respects all the metrological properties for reliable subsequent processing.

We begin our development by first considering the statistics of vision in accordance to psychophysical experiments. The Julesz conjecture establishes the idea that texture can be modeled using low-order statistics [10]. In particular, firstorder statistics (mean, variance etc.) describe the probability distribution of pixel values, while second-order statistics (cooccurrence matrix, autocorrelation etc.) consider the relation between pairs of pixel [11]. Combining both, we arrive at a spectral-spatial formulation which simultaneously characterizes the distribution of spectra and their spatial arrangement in the image. For metrological purposes, we perform the calculation of spectral difference based on the physical definition of electromagnetic spectrum. For complete texture description, we consider the resulted feature (a probability distribution) as it is without reduction to any moments.

The rest of the article is organized as follows. Section 2 details the formulation of the hyperspectral texture descriptor and its similarity measure. Section 3 describes a classification scheme to assess the feature's performance with result analysis. Section 4 presents the conclusion for this work. 


\section{PROPOSED METHOD}

We derive a hyperspectral texture descriptor that encompasses as a whole the distribution of spectra and their spatial arrangement. The formulation is based on first and second order statistics in the context of human perception. Designed to be generic, the spectral-spatial assessment based on spectral difference is adapted for any spectral range and number of bands. For compact representation, the texture feature is statistically transformed and parametrized using GMM modeling. A similarity measure is devised using Kullback-Leibler divergence.

\subsection{A spectral-spatial formulation}

For the first-order statistics $M_{1}$, we represent the spectral distribution in hyperspectral image $I$ via spectral difference $\Delta S_{r}$ of each spectrum $S$ with respect to a chosen reference $S_{r}$. Formally, $M_{1}$ is expressed as a probability distribution:

$$
M_{1}^{\left(S_{r}\right)}\left(\Delta S_{r}\right)=\operatorname{Prob}\left(d\left(S_{i}, S_{r}\right)=\Delta S_{r}\right), \forall i \in I
$$

where $d(\cdot)$ is spectral difference operator. Hence, we are able to assess the $Z$-dimensional spectral space via $z$-dimensional difference space, where $z \ll Z$. This allows us to tackle the high dimensionality of hyperspectral data without resorting to band selection or dimensionality reduction techniques. We have thus avoided changing the physical meaning of the data, therefore preserving all the metrological properties.

For the second-order statistics $M_{2}$, we refer to the work in [12] which relates co-occurrence matrix [13] with histogram of difference. On this basis, we propose to replace the cooccurrence matrix with probability distribution of spectral difference $\Delta S$ between two pixels $i$ and $j$ separated by a spatial vector $\vec{v}$. Suppose that $\vec{v}$ is defined using distance $l$ and direction $\theta, M_{2}$ is expressed as a probability distribution:

$$
\begin{aligned}
M_{2}^{(l, \theta)}(\Delta S)= & \operatorname{Prob}\left(d\left(S_{i}, S_{j}\right)=\Delta S\right), \\
& \forall i, j \in I,\|\overrightarrow{i j}\|=l, \angle \overrightarrow{i j}=\theta
\end{aligned}
$$

Combining the two statistics, we arrive at a formulation that simultaneously characterizes the distribution of spectra and their spatial arrangement in the image. Explicitly, it is expressed as a joint probability distribution $M$ given by:

$$
\begin{aligned}
& M^{\left(S_{r}, l, \theta\right)}\left(\Delta S_{r}, \Delta S\right)= \\
& \quad \operatorname{Prob}\left(d\left(S_{i}, S_{r}\right)=\Delta S_{r}, d\left(S_{i}, S_{j}\right)=\Delta S\right), \\
& \forall i, j \in I,\|\overrightarrow{i j}\|=l, \angle \overrightarrow{i j}=\theta
\end{aligned}
$$

termed Relative Spectral Difference Occurrence Matrix (RSDOM). Conceptually, it is illustrated in Figure 1 which shows the fusion of spectral and spatial analysis as a whole.

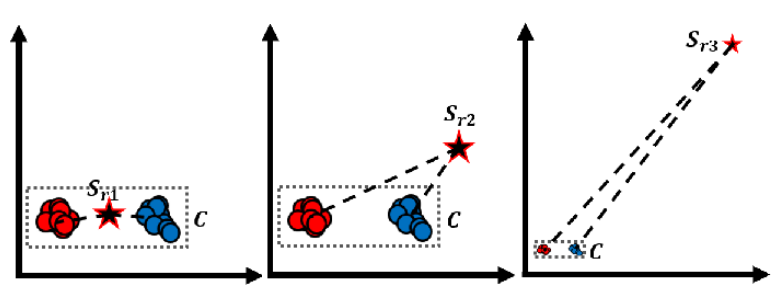

(a) $S_{r 1}$

(b) $S_{r 2}$

(c) $S_{r 3}$

Fig. 2: Representation of two different spectral distributions, $\mathbb{S}_{1}$ and $\mathbb{S}_{2}$ in difference space, with three choices of $S_{r}$.

On the choice of $S_{r}$, it has to be selected such that it lies just outside the convex hull $C$ of the spectral distribution for the entire set of texture [14]. To understand this, suppose that there are two textures to be discriminated. Figure 2 illustrates their spectral distributions $\mathbb{S}_{1}$ and $\mathbb{S}_{2}$ as well as three choices of $S_{r}$ in the difference space. $S_{r 1}$, which lies inside $C$ is a bad choice as it risks perceiving $\mathbb{S}_{1}$ and $\mathbb{S}_{2}$ as being equidistant. Lying far from $C, S_{r 2}$ on the other hand risks perceiving $\mathbb{S}_{1}$ and $\mathbb{S}_{2}$ to be equally far. $S_{r 3}$, which lies just outside $C$ is clearly the best choice as it discriminates $\mathbb{S}_{1}$ and $\mathbb{S}_{2}$ best.

\subsection{Metrological calculation of spectral difference}

A spectrum is a continuous function over the wavelength $\lambda$. Even in discretized form, its value ordering matters as there exists high correlation between neighboring spectral bands. As such, distribution-based spectral distances are more adapted for metrological purposes [15]. In this work, we choose to use Kullback-Leibler pseudo-divergence (KLPD) [16]. Given spectra $S_{1}$ and $S_{2}$, their KLPD is expressed as sum of shape difference $\Delta G$ and intensity difference $\Delta W$ :

$$
d\left(S_{1}, S_{2}\right)=\Delta G\left(S_{1}, S_{2}\right)+\Delta W\left(S_{1}, S_{2}\right)
$$

where:

$$
\begin{aligned}
& \Delta G\left(S_{1}, S_{2}\right)=k_{1} \cdot K L\left(\bar{S}_{1} \| \bar{S}_{2}\right)+k_{2} \cdot K L\left(\bar{S}_{2} \| \bar{S}_{1}\right) \\
& \Delta W\left(S_{1}, S_{2}\right)=\left(k_{1}-k_{2}\right) \log \left(\frac{k_{1}}{k_{2}}\right)
\end{aligned}
$$

noting that $K L(\cdot \| \cdot)$ is the Kullback-Leibler divergence. The normalized spectra $\bar{S}$ is expressed as:

$$
\bar{S}=\left\{\bar{s}_{j}(\lambda)=\frac{s_{j}(\lambda)}{k}, \forall \lambda \in\left[\lambda_{\min }, \lambda_{\max }\right]\right\}
$$

where the normalization constant is given by:

$$
k=\int_{\lambda_{\min }}^{\lambda_{\max }} s_{j}(\lambda) d \lambda
$$




\subsection{Parametrization and similarity measurement}

The Gaussian Mixture Model (GMM), a method for identifying mixture distributions can be used for estimating probability density functions (p.d.f.). The GMM is obtained by maximization of the likelihood function using ExpectationMaximization (EM) algorithms such as SEAM and SEM [17]. However, the mixture of $D$-dimensional multivariate normal distribution has its support confined in all the space $\mathbb{R}^{D}$, that is, it has unbounded support. The change of variable method [18] identifies the diffeomorphism adapted to the support of p.d.f. while being least rapid at infinity. It is applied on the original data and has the effect of transformation into the entire $\mathbb{R}^{D}$. Following the application of GMM, density of the original data can thus be deduced for statistical modeling.

In case of RSDOM which is defined in the $\mathbb{R}_{+}^{4}$ support by construction (due to KLPD being a positive measure), the best diffeomorphism according to [19] consists of the tensor product of the logarithm function with each variable. The transformed RSDOM is given below, where $\stackrel{\text { def }}{=} \log (\cdot)$.

$$
\begin{aligned}
& M^{\left(S_{r}, l, \theta\right)}\left(\widetilde{\Delta} G_{r}, \widetilde{\Delta} W_{r}, \widetilde{\Delta} G, \widetilde{\Delta} W\right)= \\
& \operatorname{Prob}\left(\widetilde{d}\left(S_{i}, S_{r}\right)=\left(\widetilde{\Delta} G_{r}, \widetilde{\Delta} W_{r}\right), \widetilde{d}\left(S_{i}, S_{j}\right)=(\widetilde{\Delta} G, \widetilde{\Delta} W)\right) \\
& \forall i, j \in I,\|\overrightarrow{i j}\|=l, \angle \overrightarrow{i j}=\theta
\end{aligned}
$$

As demonstration, Figure 3 depicts the 4- $D$ RSDOM in two 3- $D$ representations for the "Chili" texture from HyTexiLa. Using GMM modeling, RSDOM is finally reduced to $K \cdot(1+$ $\left.D+D^{2}\right)$ scalar values, where $K$ is the number of mixture components and $D=4$ is the number of dimension.

Considering RSDOM as probability distribution, KullbackLeibler (KL) divergence [20] is well adapted to assess the similarity between them [21]. The variational approximation of KL divergence between two GMMs is given by [22]:

$$
K L_{v a r}\left(M \| M^{\prime}\right)=\sum_{i} \phi_{i} \log \frac{\sum_{j} \phi_{j} e^{-K L\left(\mathcal{N}_{i} \| \mathcal{N}_{j}\right)}}{\sum_{j} \phi_{j}^{\prime} e^{-K L\left(\mathcal{N}_{i} \| \mathcal{N}_{j}^{\prime}\right)}}
$$

as KL divergence for $D$-dimensional normal distributions $\mathcal{N}$ is:

$$
\begin{aligned}
K L\left(\mathcal{N}_{i} \| \mathcal{N}_{j}\right)=\frac{1}{2}[ & \log \frac{\left|\Sigma_{j}\right|}{\left|\Sigma_{i}\right|}+\operatorname{tr}\left(\Sigma_{j}^{-1} \Sigma_{i}\right)-D \\
& \left.+\left(\mu_{j}-\mu_{i}\right)^{T} \Sigma_{j}^{-1}\left(\mu_{j}-\mu_{i}\right)\right]
\end{aligned}
$$

where $\phi, \mu$ and $\Sigma$ are the mixture weight, mean and covariance respectively [23]. As KL divergence is not symmetric, the similarity measure for RSDOM is defined as:

$$
d_{K L}\left(M, M^{\prime}\right)=K L_{v a r}\left(M \| M^{\prime}\right)+K L_{v a r}\left(M^{\prime} \| M\right)
$$

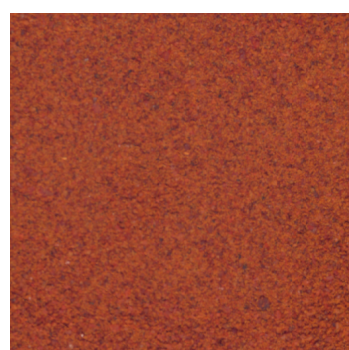

(a) “Chili”, sRGB-rendered

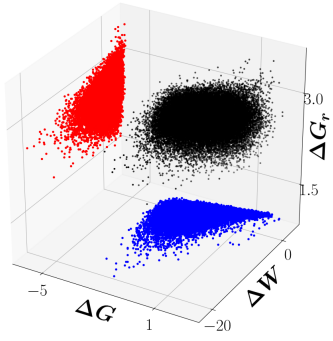

(b) RSDOM in the 3- $D$ subspace of $\Delta G-\Delta W-\Delta G_{r}$ (in black), with projection in $\Delta W-\Delta G_{r}$ (in red) and in $\Delta G-\Delta W$ (in blue)

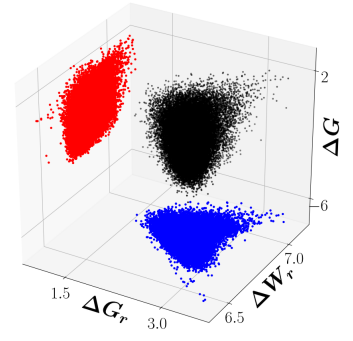

(c) RSDOM in the 3-D subspace of $\Delta G_{r}-\Delta W_{r}-\Delta G$ (in black) with projection in $\Delta W_{r}-\Delta G$ (in red) and in $\Delta G_{r}-\Delta W_{r}$ (in blue)
Fig. 3: Two 3- $D$ representations of the 4- $D$ RSDOM.

\section{EXPERIMENT AND DISCUSSION}

To assess the performance of RSDOM, we apply a classification scheme on a hyperspectral texture dataset. As comparison, we benchmark our performance against Opponent Band Local Binary Pattern (OBLBP). We annotate the results with discussion and suggestions for future improvement.

\subsection{Classification using nearest neighbor}

HyTexiLa [24] is a hyperspectral reflectance image dataset consisting of 112 textured images from five categories: food (10 images), stone (4 images), textile (65 images), vegetation (15 images) and wood (18 images). The number of spectral band is 186, ranging from $405.37 \mathrm{~nm}$ to $995.83 \mathrm{~nm}$ at $3.19 \mathrm{~nm}$ interval, hence spanning both visible and near infrared region.

The classification is based on nearest neighbor approach. Following the setting in [24], each image (class) is split into 25 patches, of which 12 is used for training and 13 for testing. To avoid any bias, we repeat all classification using $T=10$ trials with random selection of training and testing sets. We report the average accuracy (number of correct classification) and F1-score along with their standard error (standard deviation $/ \sqrt{T}$ ). As baseline comparison, we also present the result of classification based on average spectrum ${ }^{1}$.

\footnotetext{
${ }^{1}$ For an image of $N$ pixels, the average spectrum is marginally defined as $S_{\mu}(\lambda)=\left\{s_{\mu}(\lambda)=\frac{1}{N} \sum_{i=1}^{N} s(\lambda), \forall \lambda \in\left[\lambda_{\min }, \lambda_{\max }\right]\right\}$
} 


\begin{tabular}{ccccc}
\hline Classi. & \multicolumn{2}{c}{ Average spectrum } & \multicolumn{2}{c}{ RSDOM } \\
\hline & Accuracy & F1-score & Accuracy & F1-score \\
w textile & $91.4 \pm 0.3$ & $91.7 \pm 0.2$ & $95.6 \pm 0.2$ & $95.8 \pm 0.1$ \\
w/o textile & $82.2 \pm 0.5$ & $82.7 \pm 0.5$ & $92.6 \pm 0.3$ & $93.0 \pm 0.3$ \\
\hline
\end{tabular}

Table 1: Inter-categorical classification on HyTexiLa.

\begin{tabular}{ccccc}
\hline Category & \multicolumn{2}{c}{ Average spectrum } & \multicolumn{2}{c}{ RSDOM } \\
\hline & Accuracy & F1-score & Accuracy & F1-score \\
Food & $96.3 \pm 0.5$ & $96.4 \pm 0.5$ & $97.5 \pm 0.2$ & $97.7 \pm 0.2$ \\
Stone & $86.7 \pm 1.2$ & $87.4 \pm 1.1$ & $98.5 \pm 0.5$ & $98.5 \pm 0.4$ \\
Textile & $98.7 \pm 0.1$ & $98.8 \pm 0.1$ & $98.6 \pm 0.1$ & $98.6 \pm 0.1$ \\
Vegetation & $83.9 \pm 0.6$ & $84.2 \pm 0.6$ & $94.3 \pm 0.5$ & $94.4 \pm 0.5$ \\
Wood & $78.9 \pm 0.7$ & $79.3 \pm 0.4$ & $92.2 \pm 0.5$ & $92.5 \pm 0.5$ \\
\hline
\end{tabular}

Table 2: Intra-categorical classification on HyTexiLa.

In [24], OBLBP is calculated with $l=1$ on a neighborhood of 8 pixels, roughly equivalent to assessing spectral variation across three pixels. For fair comparison, we select $l=3$ in the calculation of RSDOM. For the direction, we arbitrary choose $\theta=0$. On the number of GMM components, we select $K=4$ based on the Bayesian information criterion (BIC). On the choice of $S_{r}$, we decide to adapt the rule in [14] considering the large number of image in HyTexiLa. We divide the images into two groups: the first consists of natural and complex textures (food, stone, vegetation, wood) while the second involves man-made and relatively simple textures (textile). For balanced performance, we choose to dedicate more attention on the first group. As such, we select $S_{r}$ such that it lies just outside the convex hull of the spectral distribution for the set of image belonging to the first group ${ }^{2}$.

\subsection{Results and discussion}

We perform two classifications, one on the entire dataset and the other with textile images excluded. As textile images are spectrally discriminable, excluding them from the classification allows better assessment of texture discrimination. From Table 1, it can be seen that in both cases the classification is excellent (95.6\% and 92.6\%), comparable to the accuracy obtained using OBLBP $(98.76 \%)$ in [24]. It can be observed too that RSDOM outperforms average spectrum in the baseline comparison, thus validating the interest of its formulation.

As texture complexity varies across the categories, we refer the intra-categorical classification results to try explaining the inter-categorical misclassification. From Table 2, it can be inferred that the misclassification is mainly due to vegetation and wood images. For this, we deduce three possible

\footnotetext{
${ }^{2}$ In this work, we empirically select $S_{r}(\lambda)=3(0.5+0.5 \operatorname{erf}([\lambda-$ $\widetilde{\lambda}] / 200)$, where $\operatorname{erf}(\cdot)$ is the error function and $\widetilde{\lambda}=\left(\lambda_{\max }-\lambda_{\min }\right) / 2$.
}

explanations. Firstly, the textures are mainly green for vegetation and brown for wood, therefore making discrimination from each other harder. Secondly, their texton (the smallest repeatable structure) size is larger, inducing the need for larger $l$. Thirdly, some of the textures exhibits directionality, therefore requiring a multi-direction texture assessment.

Compared to OBLBP, RSDOM registers slightly lower performance although in the same efficiency range. This can be attributed to the fact that OBLBP is a multi-directional texture descriptor as opposed to RSDOM being a uni-directional one. However, the size of RSDOM (84 scalar values with $K=4$ ) is only $0.1 \%$ of OBLBP's (82944 scalar values). Such significant difference is also reflected in the computational complexity. Considering an image of $N$ pixels, $L$ spectral bands and texture assessment in $P$ directions, the RSDOM complexity is $O(N \cdot L)$ in contrast to OBLBP's $O\left(N \cdot P \cdot 2^{L}\right)$. For each patch of $N=41616$ in the HyTexiLa classification scheme, the RSDOM computational time is about 10 seconds.

\subsection{Future work}

We choose $l=3$ for result comparison with [24], but in fact such value may be susceptible to noise. In practical, the selection should depend on the image resolution, noise and texton size. A protocol for selecting optimal $l$ value ought to be carefully developed, even in future multi-scale and multi-direction implementation. It is also worth reminded that in this work the choice of $S_{r}$ is made empirically. An optimization procedure is worth being pursued for maximized performance. On the other hand, the baseline comparison shows that the performance of classification using average spectrum alone is close to that of using RSDOM. This may be attributed to the fact that HyTexiLa is a relatively small dataset with limited spectral and spatial variety. For a more adequate evaluation of hyperspectral texture descriptors, a dataset richer in both spectral and spatial characteristics is required.

\section{CONCLUSION}

We have proposed a new hyperspectral texture descriptor named Relative Spectral Difference Occurrence Matrix (RSDOM). Being a spectral-spatial formulation, it assesses the distribution of spectra and their spatial arrangement in the image. Developed in a metrological framework to conform to physical measurements, RSDOM is adapted for any number of spectral band or range. Its calculation based on spectral difference solves the curse of dimensionality without resorting to band selection or dimensionality reduction techniques. Its efficiency has been evaluated via a texture classification scheme on HyTexiLa with excellent accuracy (95.6\%). The performance is close to Opponent Band Local Binary Pattern (OBLBP), but at a much-reduced feature size $(0.1 \%$ of OBLBP's in this work) and computational complexity. 


\section{REFERENCES}

[1] J. Transon, R. D'Andrimontand, A. Maugnard, and P. Defourny, "Survey of hyperspectral earth observation applications from space in the sentinel-2 context," Remote Sensing, vol. 10, no. 2, 2018.

[2] J. Li and R. M. Narayanan, "Integrated spectral and spatial information mining in remote sensing imagery," IEEE Trans. on Geoscience and Remote Sensing, vol. 42, no. 3, pp. 673-685, March 2004.

[3] P. Bunting, W. He, R. Zwiggelaar, and R. Lucas, Combining Texture and Hyperspectral Information for the Classification of Tree Species in Australian Savanna Woodlands, pp. 19-26, Springer, 2009.

[4] R. Casa, F. Castaldi, S. Pascucci, A. Palombo, and S. Pignatti, "A comparison of sensor resolution and calibration strategies for soil texture estimation from hyperspectral remote sensing," Geoderma, vol. 197-198, pp. $17-26$, apr 2013.

[5] X. Guo, X. Huang, and L. Zhang, "Three-dimensional wavelet texture feature extraction and classification for multi/hyperspectral imagery," IEEE Geo. and Remote Sensing Lett., vol. 11, no. 12, pp. 2183-2187, Dec 2014.

[6] S. W. Brown B. C. Johnson and J.P. Rice, "Metrology for remote sensing radiometry," in Post-Launch Calibration of Satellite Sensors, Proceedings of the International Workshop on Radiometric and Geometric Calibration, 2003, pp. 7-16.

[7] E. Honkavaara, R. Arbiol, L. Markelin, L. Martinez, M. Cramer, S. Bovet, L. Chandelier, R. Ilves, S. Klonus, P. Marshal, D. Schläpfer, M. Tabor, C. Thom, and N. Veje, "Digital airborne photogrammetry—a new tool for quantitative remote sensing?," Remote Sensing, vol. 1 , no. 3, pp. 577-605, 2009.

[8] S. W. Ng and A. Zulkifli, "Establishing metrological traceability for radiometric calibration of earth observation sensor in malaysia," IOP Conference Series: Material Sci. and Eng., vol. 152, pp. 012028, oct 2016.

[9] P. De Bièvre, "The 2012 international vocabulary of metrology: "vim"," Accreditation and Quality Assurance, vol. 17, no. 2, pp. 231-232, Apr 2012.

[10] B. Julesz, "Visual pattern discrimination," IRE Trans. on Info. Theory, vol. 8, no. 2, pp. 84-92, February 1962.

[11] D. J. Field, "What the statistics of natural images tell us about visual coding," in Human Vision, Visual Processing, and Digital Display, 1989, vol. 1077, pp. 269-276.
[12] M. Unser, "Sum and difference histograms for texture classification," IEEE Trans. on Patt. Anal. and Mac. Intel., vol. PAMI-8, no. 1, pp. 118-125, Jan 1986.

[13] R. M. Haralick, K. Shanmugam, and I. Dinstein, "Textural feature for image classification," IEEE Trans. on Systems, Man and Cybernetics, vol. 3, no. 6, pp. 610621, 1973.

[14] H. Deborah, Towards Spectral Mathematical Morphology, Theses, Université de Poitiers ; Norwegian University of science and technology, Dec. 2016.

[15] H. Deborah, N. Richard, and J. Y. Hardeberg, "A comprehensive evaluation of spectral distance functions and metrics for hyperspectral image processing," IEEE Journal of Sel. Top. in App. Earth Observations and Remote Sensing, vol. 8, no. 6, pp. 3224-3234, June 2015.

[16] N. Richard, D. Helbert, C. Olivier, and M. Tamisier, "Pseudo-Divergence and Bidimensional Histogram of Spectral Differences for Hyperspectral Image Processing," Journal of Imaging Science and Technology, 2016.

[17] G. McLachlan and T. Krishnan, The EM Algorithm and extensions, John Wiley and Sons, 2007.

[18] J. W. Osborne, "Improving your data transformations: Applying the box-cox transformation," Prac. Assess., Research and Eval., vol. 15, no. 12, pp. 1-9, 2010.

[19] S. Saoudi, F. Ghorbel, and A. Hillion, "Some statistical properties of the kernel-diffeomorphism estimator," Applied Stochastic Models and Data Analysis, vol. 13, pp. 39-58, 1997.

[20] S. Kullback and R. A. Leibler, "On info. and suff.," Ann. Math. Statist., vol. 22, no. 1, pp. 79-86, 1951.

[21] Z. Bylinskii, T. Judd, A. Oliva, A. Torralba, and F. Durand, "What do different evaluation metrics tell us about saliency models?," IEEE Trans. on Patt. Anal. and Mac. Intel., vol. 41, no. 3, pp. 740-757, March 2019.

[22] J. R. Hershey and P. A. Olsen, "Approximating the kullback leibler divergence between gaussian mixture models," in 2007 IEEE International Conference on Acoustics, Speech and Signal Processing - ICASSP '07, April 2007, vol. 4, pp. IV-317-IV-320.

[23] C. E. Rasmussen and C. K. I. Williams, Gaussian Processes for Machine Learning (Adaptive Computation and Machine Learning), The MIT Press, 2005.

[24] H. A. Khan, S. Mihoubi, B. Mathon, J. B. Thomas, and J. Y. Hardeberg, "Hytexila: High resolution visible and near infrared hyperspectral texture images," Sensors, vol. 18, no. 7, 2018. 